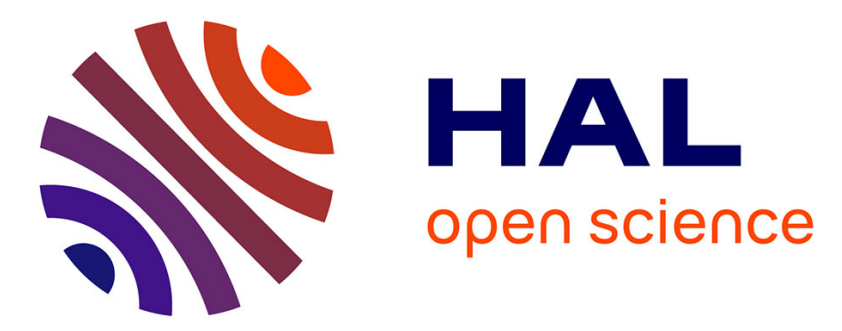

\title{
Hyperthermia reduces electromechanical delay via accelerated electrochemical processes
}

Adèle Mornas, Sébastien Racinais, Franck Brocherie, Marine Alhammoud, Robin Hager, Yanis Desmedt, Gaël Guilhem

\section{To cite this version:}

Adèle Mornas, Sébastien Racinais, Franck Brocherie, Marine Alhammoud, Robin Hager, et al.. Hyperthermia reduces electromechanical delay via accelerated electrochemical processes. Journal of Applied Physiology, 2021, 130, pp.290 - 297. 10.1152/japplphysiol.00538.2020 . hal-03466475

\section{HAL Id: hal-03466475 \\ https://hal.science/hal-03466475}

Submitted on 22 Dec 2021

HAL is a multi-disciplinary open access archive for the deposit and dissemination of scientific research documents, whether they are published or not. The documents may come from teaching and research institutions in France or abroad, or from public or private research centers.
L'archive ouverte pluridisciplinaire HAL, est destinée au dépôt et à la diffusion de documents scientifiques de niveau recherche, publiés ou non, émanant des établissements d'enseignement et de recherche français ou étrangers, des laboratoires publics ou privés. 


\section{Hyperthermia reduces electromechanical delay via accelerated \\ 2 electrochemical processes}

Running title: Heat therapy hastens muscle contraction

6 Adèle MORNAS ${ }^{1,2}$, Sébastien RACINAIS ${ }^{1,3}$, Franck BROCHERIE ${ }^{1}$, Marine

7 ALHAMMOUD $^{3}$, Robin HAGER ${ }^{1}$, Yanis DESMEDT $^{1}$, Gaël GUILHEM $^{1 *}$

$8{ }^{1}$ French Institute of Sport (INSEP), Laboratory Sport, Expertise and Performance (EA 7370),

9 Paris, France

$10 \quad{ }^{2}$ Université de Paris

$11{ }^{3}$ Aspetar Orthopaedic and Sports Medicine Hospital, Athlete Health and Performance 12 Research Centre, Doha, Qatar

14 *Correspondence and reprints:

15 Gaël Guilhem

16 Institut National du Sport, de l'Expertise et de la Performance

17 Laboratoire Sport, Expertise et Performance (EA 7370)

18 11, avenue du Tremblay

1975012 Paris

20 France

21 Tel: +33(0)141744336

22 Fax: +33(0)141754535

23 e-mail: gael.guilhem@insep.fr

Keywords: muscle temperature; contractile properties; muscle fascicle motion; force transmission 


\section{ABSTRACT}

The present study aimed to determine the effect of hyperthermia on both electrochemical and mechanical components of the electromechanical delay (EMD), using very-high-frame-rate ultrasound. Electrically evoked peak twitch force, EMD, electrochemical $\left(\mathrm{D}_{\mathrm{m}}\right.$, i.e., delay between stimulation and muscle fascicle motion) and mechanical $\left(\mathrm{T}_{\mathrm{m}}\right.$, i.e., delay between fascicle motion and force production onset) components of EMD were assessed in sixteen participants. Assessments were conducted in a control ambient environment $\left(\mathrm{CON}: 26^{\circ} \mathrm{C}\right.$, $34 \%$ relative humidity) and in hot ambient environment (HOT: $46-50^{\circ} \mathrm{C}, 18 \%$ relative humidity, after $\sim 127 \mathrm{~min}$ of heat exposure). Following heat exposure, gastrocnemius medialis temperature was $37.0 \pm 0.6^{\circ} \mathrm{C}$ in $\mathrm{HOT}$ vs. $34.0 \pm 0.8^{\circ} \mathrm{C}$ in $\mathrm{CON}(P<0.001)$. EMD was shorter $(9.4 \pm 0.8 \mathrm{~ms})$ in HOT than $\mathrm{CON}(10.8 \pm 0.6 \mathrm{~ms}, P<0.001)$. Electrochemical processes were shorter in HOT than CON $(4.0 \pm 0.8 \mathrm{~ms}$ vs. $5.5 \pm 0.9 \mathrm{~ms}$, respectively, $P<0.001)$, while mechanical processes were unchanged $(P=0.622)$. These results demonstrate that hyperthermia reduces electromechanical delay via accelerated electrochemical processes while force transmission along the active and passive parts of the series elastic component is not affected following heat exposure. The present study demonstrates that heat exposure accelerates muscle contraction thanks to faster electrochemical processes. Further investigations during voluntary contractions would contribute to better understand how these findings translate into motor performance. 
- Hyperthermia (targeted core temperature: $38.5^{\circ} \mathrm{C}$ ) reduces the time between gastrocnemius medialis stimulation and the onset of plantar flexor force production in vivo.

- This reduction in electromechanical delay is concomitant to an earlier motion of muscle fascicle compared to thermoneutral environment.

- However, hyperthermia has no impact on the duration of force transmission along aponeurosis and tendon, thereby reflecting different effects of heat exposure on contractile and elastic properties of the muscle-tendon unit. 
A rise in core or muscle temperature affects muscle metabolism $(2,14)$, neural drive $(31)$, contractile properties (10) and overall force production (33). Indeed, tissue temperature is recognized to influence mechanical and biochemical properties of skeletal muscle $(5,13)$. In vitro, a rise in temperature increases electrically-evoked rate of force development (RFD) (9, 36), with an inverse relationship between temperature and contraction time (CT; i.e., the time between the onset of force rise and peak twitch force) and half-relaxation time (HRT; i.e., the time to obtain half of the decline in twitch maximal force) inferred from a muscle twitch (41). In vivo, a rise in muscle temperature has been reported to consistently shorten $\mathrm{CT}$ and $\mathrm{HRT}$ of peak twitch, while its amplitude increased (11) or was not altered with heat $(29,32,34)$. Although these findings reflect an improvement in neuromuscular function during explosive motor tasks, the origins of this effect remain unsolved.

The ability to generate force rapidly is influenced by the neural drive transmission to the muscle, the propagation of the action potential, the excitation-contraction coupling processes and the muscle force transmission along the series elastic component $(25,28)$. On the one hand, it is well established that axonal conduction velocity increases in hot environments (38, 43). On the other hand, little is known regarding the impact of hyperthermia on force transmission efficiency by the contractile and elastic components. Diverse effects have been reported on electromechanical processes involved in force transmission efficiency in response to an increase in temperature. Heat increases ATPase activity of the myosin head (3), while it promotes calcium retention by the sarcoplasmic reticulum (42). In addition, heat may differently impact the mechanical properties of the active myofibrils (39) and the passive aponeurosis and tendon within the series elastic components (18). Because the respective contributions of contractile and elastic materials may be challenging to dissociate in vivo, 
81 further evidence is required to clarify the acute effects of heat exposure on the muscle-tendon unit components.

The measurement of time between the muscle activation onset and force production (EMD; i.e., electromechanical delay) reflects both electrochemical processes (i.e., synaptic transmission, action potential propagation through the sarcolemma, excitation-contraction coupling) and mechanical processes (i.e., force transmission along the active and passive parts of the series elastic component) (6). Thus, the investigation of EMD would provide more information about the mechanisms and muscle-tendon components involved in force transmission efficiency. EMD has been shown to be sensitive to cooling $(7,46)$. Indeed, the decrease in muscle or skin temperature, subsequent to the localized application of cold, induces an increase in EMD in humans $(21,26,46)$. Using mechanomyography, Cè et al. (7) reported a lengthening of the electrochemical but not the mechanical processes in response to muscle cooling. Using very high-frame-rate ultrasound, it is possible to detect the onset of muscle fascicle and myotendinous junction motions in response to myostimulation $(22,23$, 24, 28). This technique offers the opportunity to determine the respective contribution of electrochemical and mechanical processes to heat-induced changes in EMD in humans. Thus, the present study may contribute to better identify the steps of force production and transmission potentially responsible for the improvement in explosive strength reported in the literature following heat exposure.

The present study aimed to determine (i) the influence of hyperthermia on EMD; and (ii) the respective heat-mediated alterations in electrochemical and/or force transmission processes. We hypothesized that EMD would decrease in response to heat exposure likely due to faster electrochemical processes. 


\section{Participants}

108 Sixteen recreationally active participants (9 men and 7 women, age: $24.9 \pm 5.7$ yrs, height:

$109174.8 \pm 7.5 \mathrm{~cm}$ and body mass: $69.8 \pm 9.9 \mathrm{~kg}$ ), with no recent history of ankle disorder or 110 injury, participated in this study. Participants completed a pre-inclusion medical visit 111 consisting of a clinical examination, a medical history questionnaire and anthropometric 112 measurements before entering to the study. They were informed regarding the nature, aims 113 and risks associated with the experimental procedures before providing written consent. This 114 study was approved by the Sud-Ouest et Outre-mer III ethics committee (approval reference: 115 3849, ID-RCB: 2019-A00596-51) and conformed to the standards of the Declaration of 116 Helsinki.

\section{Experimental design}

119 Two to four days after a familiarization session allowing the participants to be accustomed to 120 the procedures, the participants performed two identical testing sequences during a single 121 visit, including electrically-evoked peak twitch and EMD assessment. The tests were first 122 performed in control ambient environment $\left(\mathrm{CON} ; 25.8 \pm 1.8^{\circ} \mathrm{C}, 33.6 \pm 8.6 \%\right.$ relative 123 humidity) and then in hot ambient environment (HOT; $47.4 \pm 1.84^{\circ} \mathrm{C}, 18.5 \pm 4.7 \%$ relative 124 humidity) after the participants passively reached a core temperature of $38.5^{\circ} \mathrm{C}$ (i.e., after 127 $125 \pm 33 \mathrm{~min}$ from the onset of heat exposure). After heat exposure, muscle temperature increased 126 from $34.0 \pm 0.8^{\circ} \mathrm{C}$ to $37.0 \pm 0.6^{\circ} \mathrm{C}(P<0.001)$ in a subsample of six participants. The 127 hyperthermia was controlled by an environmental chamber (Thermo-training room, Paris, 
128 France) and participants could drink at libitum. Body mass was measured before and after

129 heat exposure to evaluate the weight loss due to dehydration.

\section{Twitch force}

132 A custom-built ergometer composed of a specific footplate (Bio2M, Compiègne, France) was used to measure plantar flexor force evoked by electrical nerve stimulations. Participants were laid prone with their legs fully extended and their ankle flexed at $90^{\circ}$ (i.e., foot perpendicular to the tibia). Their right foot was firmly fixed on the footplate connected to a force sensor (2712-100 daN-0.02-B, Sensy, Charleroi, Belgium). The force signal was digitized at a sampling rate of $2 \mathrm{kHz}$ using an analog-to-digital converter designed in our laboratory. The tibial nerve was electrically stimulated using a constant current stimulator (Digitimer DS7AH, Digitimer, Letchworth Garden City, UK) delivering a single electrical pulse (1000 $\mu \mathrm{s}, 400 \mathrm{~V})$ through a cathode placed in the popliteal cavity and an anode placed distally to the patella. The intensity was adjusted for each participant by progressive increase in current (i.e., incremental step of $10 \mathrm{~mA}$ ) until plantar flexor force reached a plateau. Thereafter, five stimulations were delivered at the electrical intensity required to elicit peak force multiplied by 1.5 .

Participants laid prone with their legs fully extended on a second home-made ergometer previously used to measure the EMD on plantar flexor muscles $(22,23,28)$. Their right foot was firmly attached in a rigid cycling shoe (chosen to avoid possible dynamics in coupling between the shoe and the force sensor) fixed on an adjustable system connected to a force 
152 Percutaneous electrical stimulations were applied over the gastrocnemius medialis (GM) to 153 elicit contraction by a single electrical pulse $(1000 \mu \mathrm{s}, 400 \mathrm{~V})$ through two electrodes, one 154 placed on the motor point (previously determined as the location inducing the strongest twitch 155 with a low electrical stimulation) and the other placed on the distal portion of GM. The 156 stimulation intensity corresponded to the intensity necessary to obtain peak force. An ultrafast 157 ultrasound scanner (v. 12, Aixplorer, Supersonic Imagine, Aix en Provence, France) coupled 158 with a linear transducer array (4-15 MHz, SuperLinear 15-4, Vermon, Tours, France) was 159 used to acquire raw radiofrequency signals at $4 \mathrm{kHz}$. Muscle stimulations were delivered 50 $160 \mathrm{~ms}$ after the onset of ultrasound images acquisition through an automatized trigger signal 161 transmitted from the ultrasound scanner to the stimulator, thereby ensuring a consistent 162 synchronization between mechanical and ultrasound data. For each participant, two bouts 163 (muscle and tendon trials) composed of three electrically-evoked contractions with 1 min rest 164 between each trial were performed. During the muscle trials, the ultrasound probe was placed 165 over the GM muscle belly to detect the onset of muscle fascicle motion, reflective of electrochemical processes. During tendon trials, the ultrasound probe was maintained over the distal myotendinous junction of GM to detect the onset of myotendinous junction motion, reflective of mechanical processes. These two bouts were performed alternating the order, which was the same in CON and HOT. Participants were instructed to fully relax before each 170 stimulation.

\section{Temperature monitoring}

173 Prior to testing, participants were instrumented to control their core and skin temperatures.

174 Core temperature was monitored rectally using an electronic capsule (e-Celcius, BodyCap, 175 Caen, France) self-inserted by the length of a gloved finger. Participants were instrumented 
176 with four data loggers (iButtons, Maxim Integrated, USA) measuring skin temperature at the

177 arm, chest, thigh and shin to calculate the average skin temperature, according to the 178 following equation (35):

$0.3 \times$ chest temperature $+0.3 \times$ arm temperature $+0.2 \times$ thigh temperature $+0.2 \times$ shin temperature

179 Core and skin temperatures were continuously measured throughout the testing sequences and

180 heat exposure. To obtain temperatures values in HOT, the mean value of core and skin

181 temperatures were averaged during the testing sequence after heat exposure. In addition,

182 muscle temperature of contralateral GM was measured immediately before each testing 183 sequence in a subsample of six participants using a needle intramuscular thermistor 184 (MKA08050-A, Ellab, Roedovre, Denmark) inserted under local anesthesia after skin 185 disinfection.

\section{Data analysis}

188 Data were analysed using custom-written scripts (Origin 2020, OriginLab corporation, USA and Matlab 2010a-2017b, The Mathworks, Natick, MA).

191 First, force signal was low-pass filtered $\left(20 \mathrm{~Hz}\right.$, zero lag $3^{\text {rd }}$ order Butterworth). Then, the 192 mechanical response to the five electrically-evoked stimulations were analysed and averaged 193 to determine peak twitch amplitude (PT; i.e., the highest value of twitch force production), CT 194 and HRT. The average RFD was calculated as PT/CT (34).

196 Force signals were low-pass filtered $\left(200 \mathrm{~Hz}\right.$, zero lag $3^{\text {rd }}$ order Butterworth). We defined the 197 EMD as the delay between electrical stimulation and the force production onset. For each 
participant, six values of EMD were obtained in each test sequence: three muscle trials and

199 three tendon trials. As previously described by Nordez et al. (28) and Lacourpaille et al. (23),

200 ultrasound B-mode images were used to determine the region of interest for each stimulation

201 in order to detect the motion onset for GM fascicle during muscle trials (i.e., between the two

202 aponeuroses) and myotendinous junction during tendon trials (i.e., on the myotendinous; Fig.

203 1). The displacements of the regions of interest along the ultrasound beam axis were

204 calculated using a one-dimensional cross correlation of the windows of consecutive

205 ultrasound images. Thus, the tissue motion between two consecutive images (i.e., particle

206 velocity) was measured with micrometric precision. Absolute particle velocities were

207 averaged within previously determined regions of interest and then used to detect the onset of

208 GM fascicle (Fig. 1A) and myotendinous junction (Fig. 1B) motion. The onset of motion was

209 detected as the first point with a negative derivative of tissue motion in the reverse direction

210 time (28). The same method was used to automatically detect the onset of the force

211 production over time. Visual inspection was performed to check and validate the onset

212 detection for each signal. Then we determined the delay (in $\mathrm{ms}$ ) between the onset of

213 electrical stimulation (which corresponded to the trigger output signal collected from the

214 electrical stimulator) and either the onset of muscle fascicle motion $\left(\mathrm{D}_{\mathrm{m}}\right.$, for muscle fascicle

215 trials) or the onset of myotendinous junction motion ( $D_{t}$, for tendon trials). The delay between

216 the onset of GM fascicle motion and the onset of force production is attributed to the force

217 transmission ( $T_{m}$, time delay to force transmission). The difference between $D_{m}$ and $D_{t}$ and

218 between $\mathrm{D}_{\mathrm{t}}$ and EMD were calculated to respectively compute the delay of force transmission

219 along aponeurosis and tendon. Using the same technique as described elsewhere, Nordez et al.

220 (28) and Lacourpaille et al. (24) respectively demonstrated a good repeatability [standard

221 error measurement $(\mathrm{SEM})<0.88 \mathrm{~ms}$; coefficient of variation $(\mathrm{CV}): 5.0-11.6 \%$ ] and a good

222 inter-day reliability of EMD, $\mathrm{D}_{\mathrm{m}}$ and $\mathrm{D}_{\mathrm{t}}(\mathrm{SEM}<0.79 \mathrm{~ms} ; \mathrm{CV}: 6.8-12.5 \%)$. In the current 
study, SEM were $<0.40 \mathrm{~ms}$ and $\mathrm{CV}$ values ranged from 8.2 to $15.2 \%$ across $\mathrm{EMD}, \mathrm{D}_{\mathrm{m}}$ and

$224 \mathrm{D}_{\mathrm{t}}$.

\section{Statistical analysis}

227 All statistical analyses were performed with Statistica (v. 13.0; StatSoft, Tulsa, Oklahoma, 228 USA). Values were therefore reported as mean \pm standard deviation (SD). The assumptions of 229 normality of the data were verified using a Shapiro-Wilk's test. Paired t-tests were used to 230 compare the effect of the environmental condition (CON vs. HOT) on core, skin and muscle 231 temperatures, mechanical responses to the electrically-evoked stimulation (PT, CT, HRT and 232 RFD) and EMD. A two-way analysis of variance (ANOVA) for repeated measures [condition

$233(\mathrm{CON}, \mathrm{HOT}) \times$ delay $\left.\left(\mathrm{D}_{\mathrm{m}}, \mathrm{T}_{\mathrm{m}}\right)\right]$ was used to test whether heat exposure affected, absolute (in $234 \mathrm{~ms}$ ) and relative (in \% of EMD) values of $\mathrm{D}_{\mathrm{m}}$ and $\mathrm{T}_{\mathrm{m}}$. Then, a two-way ANOVA for repeated 235 measures [condition $(\mathrm{CON}, \mathrm{HOT}) \times$ delay (aponeurosis, tendon)] was used to test the effect of 236 heat exposure on force transmission independently of electrochemical process, in absolute and 237 relative values. When the sphericity assumption in repeated measures ANOVAs was violated 238 (Mauchly's test), a Geisser-Greenhouse correction was used. When appropriate, post hoc 239 analyses were performed using Bonferroni tests. Effect sizes were described in terms of 240 partial eta-squared $\left(\eta_{p}^{2}\right.$, with $\eta_{p}^{2} \geq 0.06$ representing a moderate effect and $\eta_{p}^{2} \geq 0.14$ a large 241 effect). Statistical significance was set as $P<0.05$.

\section{RESULTS}

\section{Heat exposure}


Mean core and skin temperatures were significantly higher in $\mathrm{HOT}\left(38.4 \pm 0.3^{\circ} \mathrm{C}\right.$ and $38.8 \pm$ $0.4^{\circ} \mathrm{C}$, respectively) than $\mathrm{CON}\left(37.0 \pm 0.3^{\circ} \mathrm{C}\right.$ and $34.7 \pm 0.7^{\circ} \mathrm{C}$, respectively; all $P$ values < 0.001). Shin skin temperature was evenly significantly higher in HOT $\left(38.8 \pm 0.9^{\circ} \mathrm{C}\right)$ than $\mathrm{CON}\left(34.3 \pm 0.9^{\circ} \mathrm{C} ; P<0.01\right)$. Muscle temperature measured in a subsample $(\mathrm{n}=6)$ from the 16 participants was $37.0 \pm 0.6^{\circ} \mathrm{C}$ in $\mathrm{HOT}$ vs. $34.0 \pm 0.8^{\circ} \mathrm{C}$ in $\mathrm{CON}(P<0.001)$. Heat exposure, which lasted $127 \pm 33 \mathrm{~min}$, had no statistical effect on body mass measured in HOT vs. $\operatorname{CON}(P=0.89)$.

\section{Twitch force}

No difference was found in PT amplitude between HOT and CON $\left(P=0.301, \eta^{2}{ }_{p}=0.036\right.$; Table 1). Both CT and HRT were shorter in HOT than CON $(-9.0 \pm 6.5 \%$ and $-15.1 \pm 10.1 \%$ respectively; $P<0.001, \eta_{\mathrm{p}}^{2} \geq 0.419$; Table 1$)$. RFD was higher in HOT than CON $(+16.9 \pm$ $32.8 \% ; P=0.029, \eta_{\mathrm{p}}^{2}=0.149 ;$ Table 1$)$

\section{EMD}

EMD was $1.37 \pm 0.87 \mathrm{~ms}(-12.6 \pm 7.7 \%)$ shorter in HOT than $\mathrm{CON}\left(P<0.001, \eta_{\mathrm{p}}^{2}=0.523\right.$; Fig. 1). This reduction in EMD consistently occurred for all participants (range: -0.08 to -2.98 ms). We observed an effect of heat exposure on absolute $\mathrm{D}_{\mathrm{m}}$ and $\mathrm{T}_{\mathrm{m}}$ (condition effect: $P<$ $0.001, \eta_{\mathrm{p}}^{2}=0.509$ ). Post hoc analysis revealed that $\mathrm{D}_{\mathrm{m}}$ was significantly shorter in HOT than $\operatorname{CON}\left(-26.5 \pm 18.2 \%, P<0.001, \eta_{\mathrm{p}}^{2}=0.416\right.$; Figs. $\left.1-2\right)$, whereas no significant difference was found for $\mathrm{T}_{\mathrm{m}}$ between HOT and CON $\left(P=0.622, \eta_{\mathrm{p}}^{2}=0.008\right.$; Fig. 1$)$. Two-way ANOVA revealed a main effect of hyperthermia on relative contribution of $D_{m}$ and $T_{m}$ to EMD $(P=$ $\left.0.015, \eta_{p}^{2}=0.182\right)$. Post hoc analysis showed that the relative contribution of $\mathrm{D}_{\mathrm{m}}$ to EMD was smaller in HOT than $\mathrm{CON}\left(-8.0 \pm 11.1 \%, P=0.015, \eta_{\mathrm{p}}^{2}=0.182\right)$. Conversely, the relative 
contribution of $\mathrm{T}_{\mathrm{m}}$ to $\mathrm{EMD}$ was higher in HOT than $\mathrm{CON}\left(+8.0 \pm 11.1 \%, P=0.015, \eta_{\mathrm{p}}^{2}=\right.$ 0.182). We found no significant main effect of heat exposure on absolute force transmission delays (aponeurosis and tendon; $P=0.270, \eta_{\mathrm{p}}^{2}=0.086$ ). However, two-way ANOVA revealed a main effect of hyperthermia on relative contribution of force transmission to EMD (aponeurosis and tendon, $P=0.034, \eta_{\mathrm{p}}^{2}=0.208$ ). Post hoc showed that the relative contribution of force transmission along aponeurosis to EMD was not altered by heat exposure $\left(P=0.294, \eta_{\mathrm{p}}^{2}=0.037\right)$, whereas relative contribution of force transmission along tendon to EMD was greater in HOT than $\mathrm{CON}\left(+10.8 \pm 11.5 \%, P=0.012, \eta^{2}{ }_{\mathrm{p}}=0.192\right)$.

\section{DISCUSSION}

The aim of this study was to determine the influence of hyperthermia induced by heat exposure on EMD and the subsequent alterations in electrochemical and mechanical processes involved in EMD. In accordance with our initial hypothesis, hyperthermia reduced the delay between muscle stimulation and the onset of plantar flexor force production in vivo. This reduction was concomitant with shorter electrochemical processes (i.e., decreased delay between muscle stimulation and the onset of muscle fascicle motion) compared with control conditions. The delay between muscle fascicle motion and force production was unchanged in hot environment, reflecting no impact of hyperthermia on force transmission along elastic components.

In line with previous reports, contraction time and half-relaxation time were shortened and rate of force development increased after heat exposure $(11,34)$. In comparison with Racinais et al. (34), the smaller reduction in contraction time (-9.0\% vs. $-36.5 \%)$ and half relaxation time $(-15.1 \%$ vs. $-25.2 \%)$ and the smaller increase in rate of force development $(+16.9 \%$ vs. $+64.7 \%$ ) observed in the current study was probably due to the smaller amplitude of core 
temperature increase with heat exposure $\left(+1.4\right.$ vs. $\left.+2.6^{\circ} \mathrm{C}\right)$. The main aim of the present experimental protocol was to identify the phases of force production and transmission that could account for this heat-mediated improved explosive performance.

The delay between muscle stimulation and the onset of force production measured under thermoneutral environment $(10.8 \pm 0.6 \mathrm{~ms})$ was in the range of EMD reported in the literature on plantar flexors muscles [i.e., $7.9 \mathrm{~ms}$ to $18.8 \mathrm{~ms}(16,17,27,28)]$. With muscle temperature increase, EMD consistently decreased in all participants by $1.37 \pm 0.87 \mathrm{~ms}$ on average (i.e., $12.6 \pm 7.7 \%$ ) as compared to the values obtained in a thermoneutral environment. In line with the present findings, a previous study reported a $16 \%$ decrease in EMD in malignant hyperthermia susceptible participants [i.e., $+4.3 \%$ of muscle temperature (2)]. Conversely, Kubo et al. (21) observed an increase in EMD of plantar flexors after a 30 min immersion in hot water of the lower limbs. The partial immersion, the difference in heat medium (water vs. ambient air) or the contraction modality and the shorter duration of heat application compared to the present study (i.e., 30 min vs. 127 min) may partly explain this discrepancy, although it is not possible to attest the impact of hot water immersion on core or muscle temperature in the aforementioned study. Using local application of heat (i.e., hot packs), Zhou et al. (46) measured EMD during voluntary contractions of quadriceps muscle at muscle temperatures between $30^{\circ} \mathrm{C}$ and $38^{\circ} \mathrm{C}$. EMD decreased from $30^{\circ} \mathrm{C}$ to $36^{\circ} \mathrm{C}$, then it increased up to $36-38^{\circ} \mathrm{C}$.

311 Our results showed a decrease in EMD from a muscle temperature of $34^{\circ} \mathrm{C}$ in thermoneutral 312 environment to $37^{\circ} \mathrm{C}$ in hot environment, suggesting that the impact of heat on EMD may 313 differ between voluntary and electrically-evoked contraction and between muscle groups. 314 Given the different properties between knee extensor and plantar flexor muscles, these 315 findings may also illustrate a possible muscle-dependent effect of heat exposure $(18,34,39)$. 316 Overall, our findings merged with previous works collectively suggest a continuum of 317 temperature-mediated effect on EMD in vivo, reflected by a negative relationship between 
muscle temperature and EMD. Further investigations exploring EMD under various muscle

319 temperatures are required to conclude with certainty on the nature of this potential relation.

320 By coupling ultrafast ultrasound to EMD assessments, we aimed to distinguish the 321 contribution of the electrochemical processes from the muscle force transmission along the 322 series elastic component to EMD. In line with our hypothesis, we observed substantial 323 differences in the delay between muscle stimulation and the onset of muscle fascicle motion 324 in hot compared to thermoneutral environment $(-1.46 \pm 1.12 \mathrm{~ms},-26.5 \pm 18.2 \%)$. This 325 reduction could be attributed to faster synaptic transmission, propagation of action potential 326 through the sarcolemma and/or excitation-contraction coupling $(6,8,28)$. Interestingly, when normalized to EMD, the relative contribution of electrochemical processes also decreased with hyperthermia $(-8.0 \pm 11.1 \%)$, demonstrating that the electrochemical processes were accelerated in a greater extent than the impact appraised on the global EMD. At a muscle 330 temperature of $23^{\circ} \mathrm{C}$ achieved through localized cold application, Cè et al. (7) reported a 331 lengthening of electrochemical processes which were multiplied by $\sim 1.5$. These variations in 332 the duration of electrochemical processes upon hot and cold environments could be related to temperature-mediated effects on axonal conduction velocity. Indeed, axonal conduction velocity can decrease with cold and inversely increase with heat $(12,38)$. On the one hand, a 335 decrease in muscle temperature is recognized to slow down both the opening and closing of $336 \mathrm{Na}^{+}$channels, while an increase in muscle temperature accelerates synaptic transmission (33, 337 37). However, the depolarization of muscle fibres depends on the influx of $\mathrm{Na}^{+}$ions through 338 cell membrane. Increasing ambient temperature reduces the amplitude and the duration of 339 depolarization, due to reduced $\mathrm{Na}^{+}$influx. This effect in turn challenges the production of a 340 muscle fibre action potential (37), which could suggest an increase in the duration of 341 electrochemical processes with heat. On the other hand, an increase in ambient temperature 342 additionally increases ATPase activity of myosin heavy chains (3) and reduces $\mathrm{Ca}^{2+}$ uptake in 
the sarcoplasmic reticulum, thereby increasing the rate of cross-bridge cycling $(40,42)$. Thus,

344 literature reports heat-mediated effects that would inversely impact the duration of

345 electrochemical processes after a heat exposure. The present experimental approach does not

346 allow to dissociate the respective contribution of each process involved in hyperthermia-

347 induced changes. However, Nordez et al. (28) suggested a minor role of the active part of the

348 series elastic component to electrochemical processes. It is therefore likely that synaptic

349 transmission and excitation-contraction coupling may be primarily affected by hyperthermia.

350 One could note that an acceleration of synaptic transmission is associated with a lower

351 amplitude of action potential, which could impact the action potential response (37). A faster

352 synaptic transmission would reduce electrochemical processes duration, which has yet been

353 shown to be associated with a lower amplitude of the action potential response with heat.

354 Hyperthermia could thus likely reduce the amplitude of the action potential. However, our

355 results showed a faster response.

356 Our findings showed that absolute delay for force transmission along the aponeurosis and the

357 tendon was not affected by ambient temperature. In their systematic review, Bleakley et al. (4)

358 reported an equivocal effect of heat or cold on passive stiffness in soft tissues. In vitro, tendon

359 viscosity and temperature follow an exponential relationship (18), suggesting an increase in

360 tendon compliance following heat exposure, confirmed by animal studies $(44,45)$ reporting a

361 greater viscous mechanical behavior with heat. In vivo, local application of heat on lower

362 limbs does not change the stiffness of tendinous tissues, assessed in passive and active

363 conditions, despite an extended range of motion $(15,21)$. Such effect of hyperthermia on the

364 mechanical properties of the series elastic component could impair the effectiveness of force

365 transmission. In contrast, cooling resulted in increasing tendon stiffness, in vitro (19) and in

366 vivo after thirty minutes of local icing (1) or four sets of four minutes, with one minute

367 recovery in between, of cryotherapy (30). These results suggest that absolute force 
transmission processes are unchanged with heat. In accordance with the current scientific literature, this advocates that tendinous stiffness could thus be unchanged or slightly diminished with heat.

\section{Methological considerations}

Our findings showed a decrease in electro-induced EMD with hyperthermia. Given that viscoelastic responses of muscle and tendon may differ between electro-induced and voluntary contractions, these results could be slightly different during voluntary contractions. However, one may assume that EMD measurements during voluntary contraction also present some drawbacks. As suggested by Hug et al. (20), EMD measurements obtained during voluntary contractions could be influenced by methodological artefacts and should be interpreted with caution. Indeed, EMD measured during voluntary contraction, with the use of electromyography, is insufficient to precisely detect the onset of activation of the studied muscle fibres. While the assessment of EMD is challenging in voluntary conditions, voluntary RFD has been shown to increase in hot environment. While electromechanical processes appeared to be the main responsible for the reduction in EMD in vivo, the relative contributions of the synaptic transmission, the excitation-contraction coupling, and the active part of the series elastic component could not be directly quantified in the present study. Further assessments of EMD and its various components in several cold and hot conditions may contribute to determine the nature of the relationships between temperature and EMD suggested in the literature (46). Given that EMD plays a major role in explosive motor task performance, the present findings demonstrate that ambient temperature should be reported with explosive strength evaluations and EMD delays measurements. The present study allowed us to locate the phases of force production and transmission potentially altered 
392 following heat exposure which could impact explosive strength and in turn sport performance.

393 With an electromechanical delay reduction of $1.37 \mathrm{~ms}$ (i.e., $12.6 \%$ of EMD in GM muscle),

394 the effect of hyperthermia can be important for performance and make a difference in many

395 sports. Further investigations during voluntary contractions are warranted to improve our

396 understanding of the effects of heat exposure on the different components of the muscle-

397 tendon unit in hot conditions and their consequences on motor performance.

\section{Conclusion}

400

401

402

403

404

405

406

407

408

409

410

411

412

413

The present study showed that hyperthermia decreased the time elapsed between the onset of muscle activation and force production, mainly through an acceleration of electrochemical processes. However, mechanical processes involved in force transmission during electromechanical delay were unchanged following heat exposure. These findings contribute to elucidate the mechanisms involved in the enhanced contractile properties previously reported following an acute heat exposure. While future research is required to further describe the role of muscle-tendon dynamics in heat-mediated effects, the present results suggest a major role of synaptic transmission and excitation-contraction coupling in electromechanical delay shortening. This study allowed to determine the respective contributions of electromechanical and mechanical processes to heat-induced changes in EMD. A shortening of electromechanical delay and electrochemical processes might explain the improvement in explosive strength reported in the literature. Future investigations of muscle-tendon dynamics involved during voluntary contractions are warranted for a better understanding of motor performance following hyperthermia. 
415 The authors are grateful to Lilian Lacourpaille (University of Nantes, France) for his support 416 in electromechanical delay measurements. The authors thank the participants for their 417 commitment.

\section{GRANTS}

420

421

422

A. Mornas is supported by a scholarship funded by the French Ministry of Research. S. Racinais was supported by a grant from Aspire Zone Foundation for this project. The Laboratory Sport, Expertise and Performance is a partner of the French network ReFORM, recognized as a Research Centre for the Prevention of Injury and Illness and the Protection of Athletes by the International Olympic Committee (IOC). As a member of the IOC Medical Research Network, ReFORM has received funding from the IOC to establish long-term research programmes on the prevention of injuries and illnesses in sport for the protection of athlete health.

\section{DISCLOSURES}

No conflicts of interest, financial or otherwise, are declared by the authors.

\section{AUTHOR CONTRIBUTIONS}

A.M., S.R., F.B. and G.G. conceived and designed the research; A.M., S.R., F.B., M.A., R.H., Y.D. and G.G. performed the experiments; A.M., R.H. and G.G. analysed the data; A.M., S.R., F.B., and G.G. interpreted the results; A.M. S.R., F.B. and G.G. prepared the figures; A.M. and G.G. drafted the manuscript; A.M., S.R., F.B., M.A. and G.G. edited and revised the manuscript; A.M., S.R., F.B., M.A., R.H., Y.D. and G.G. reviewed and approved the final version of the manuscript. 
441 Figure 1. Ultrasound images showing the regions of interest delineated with white squares for the GM

442 muscle (A) and myotendinous junction (B). Representation of the electromechanical delay (EMD) and

443 its components in control ambient (CON) and hot ambient (HOT) environment (C). Horizontal stacked

444 bar plots represent means $\pm \mathrm{SD}$. The delay between electrical muscle stimulation and the onset of 445 muscle fascicle motion is attributed to electrochemical processes $\left(D_{m}\right)$. The delay between the onset of 446 fascicle motion and the onset of force production is attributed to force transmission $\left(\mathrm{T}_{\mathrm{m}}\right)$. The delay 447 between muscle electrical stimulation and the onset of myotendinous junction motion $\left(D_{t}\right)$ reflects the 448 time required for electrochemical processes and force transmission along aponeurosis. ${ }^{\mathrm{a}}$ and ${ }^{\mathrm{b}}$ : 449 Significant difference between HOT and CON for EMD and $\mathrm{D}_{\mathrm{m}}(\mathrm{ms}) ; P<0.001$.

451 Figure 2. Delay between the onset of electrical stimulation and the onset of muscle fascicle motion $452\left(\mathrm{D}_{\mathrm{m}}, \mathrm{A}\right)$ and delay between the onset of muscle fascicle motion and the onset of force production $\left(\mathrm{T}_{\mathrm{m}}\right.$, 453 B), obtained in control ambient $(\mathrm{CON})$ and hot ambient (HOT) environment. The bold traces represent 454 the mean change, and box charts correspond to standard deviation. *** Significant difference between 455 HOT and CON, $P<0.001$. 
1. Alegre LM, Hasler M, Wenger S, Nachbauer W, and Csapo R. Does knee joint cooling change in vivo patellar tendon mechanical properties? Eur J Appl Physiol 116: 1921-1929, 2016.

2. Bäckman E, Lennmarken C, Rutberg H, and Henriksson KG. Skeletal muscle contraction characteristics in vivo in malignant hyperthermia susceptible subjects. Acta Neurol Scand 77: 278-282, 1988.

3. Bárány M. ATPase activity of myosin correlated with speed of muscle shortening. J Gen Physiol 50: Suppl:197-218, 1967.

4. Bleakley CM and Costello JT. Do thermal agents affect range of movement and mechanical properties in soft tissues? A systematic review. Arch Phys Med Rehabil 94: 149-163, 2013.

5. Bottinelli R, Canepari M, Pellegrino MA, and Reggiani C. Force-velocity properties of human skeletal muscle fibres: myosin heavy chain isoform and temperature dependence. $J$ Physiol 495 ( Pt 2): 573-586, 1996.

6. Cavanagh PR and Komi PV. Electromechanical delay in human skeletal muscle under concentric and eccentric contractions. Eur J Appl Physiol Occup Physiol 42: 159-163, 1979.

7. Cè E, Rampichini S, Agnello L, Limonta E, Veicsteinas A, and Esposito F. Effects of temperature and fatigue on the electromechanical delay components. Muscle Nerve 47: 566576, 2013.

8. Chen HY, Liau JJ, Wang CL, Lai HJ, and Jan MH. A novel method for measuring electromechanical delay of the vastus medialis obliquus and vastus lateralis. Ultrasound Med Biol 35: 14-20, 2009.

9. Close R and Hoh JF. Influence of temperature on isometric contractions of rat skeletal muscles. Nature 217: 1179-1180, 1968.

10. Davies CT and Young K. Effect of temperature on the contractile properties and muscle power of triceps surae in humans. J Appl Physiol Respir Environ Exerc Physiol 55: 191-195, 1983.

11. de Ruiter CJ, Jones DA, Sargeant AJ, and de Haan A. Temperature effect on the rates of isometric force development and relaxation in the fresh and fatigued human adductor pollicis muscle. Exp Physiol 84: 1137-1150, 1999.

12. Denton A, Bunn L, Hough A, Bugmann G, and Marsden J. Superficial warming and cooling of the leg affects walking speed and neuromuscular impairments in people with spastic paraparesis. Ann Phys Rehabil Med 59: 326-332, 2016.

13. Edman KA. The velocity of unloaded shortening and its relation to sarcomere length and isometric force in vertebrate muscle fibres. J Physiol 291: 143-159, 1979.

14. Febbraio MA, Snow RJ, Stathis CG, Hargreaves M, and Carey MF. Effect of heat stress on muscle energy metabolism during exercise. J Appl Physiol (1985) 77: 2827-2831, 1994.

15. Fujita K, Nakamura M, Umegaki H, Kobayashi T, Nishishita S, Tanaka H, Ibuki S, and Ichihashi N. Effects of a Thermal Agent and Physical Activity on Muscle Tendon Stiffness, as Well as the Effects Combined With Static Stretching. J Sport Rehabil 27: 66-72, 2018.

16. Grosset J-F, Piscione J, Lambertz D, and Pérot C. Paired changes in electromechanical delay and musculo-tendinous stiffness after endurance or plyometric training. European Journal of Applied Physiology 105: 131, 2008.

17. Hopkins JT, Feland JB, and Hunter I. A comparison of voluntary and involuntary measures of electromechanical delay. Int J Neurosci 117: 597-604, 2007.

18. Huang CY, Wang VM, Flatow EL, and Mow VC. Temperature-dependent viscoelastic properties of the human supraspinatus tendon. J Biomech 42: 546-549, 2009.

19. Huang $\mathbf{H}$, Zhang J, Sun K, Zhang $\mathbf{X}$, and Tian $\mathbf{S}$. Effects of repetitive multiple freeze-thaw cycles on the biomechanical properties of human flexor digitorum superficialis and flexor pollicis longus tendons. Clin Biomech (Bristol, Avon) 26: 419-423, 2011.

20. Hug F, Lacourpaille L, and Nordez A. Electromechanical delay measured during a voluntary contraction should be interpreted with caution. Muscle Nerve 44: 838-839, 2011. 
21. Kubo K, Kanehisa H, and Fukunaga T. Effects of cold and hot water immersion on the mechanical properties of human muscle and tendon in vivo. Clin Biomech (Bristol, Avon) 20 : 291-300, 2005.

22. Lacourpaille L, Gross R, Hug F, Guével A, Péréon Y, Magot A, Hogrel JY, and Nordez A. Effects of Duchenne muscular dystrophy on muscle stiffness and response to electricallyinduced muscle contraction: A 12-month follow-up. Neuromuscul Disord 27: 214-220, 2017.

23. Lacourpaille L, Nordez A, Doguet V, Hug F, and Guilhem G. Effect of damaging exercise on electromechanical delay. Muscle Nerve 54: 136-141, 2016.

24. Lacourpaille L, Nordez A, and Hug F. Influence of stimulus intensity on electromechanical delay and its mechanisms. J Electromyogr Kinesiol 23: 51-55, 2013.

25. Maffiuletti NA, Aagaard P, Blazevich AJ, Folland J, Tillin N, and Duchateau J. Rate of force development: physiological and methodological considerations. Eur J Appl Physiol 116: 1091-1116, 2016.

26. Mallette MM, Cheung SS, Kumar RI, Hodges GJ, Holmes MWR, and Gabriel DA. The effects of local forearm heating and cooling on motor unit properties during submaximal contractions. Exp Physiol, 2020.

27. Muraoka T, Muramatsu T, Fukunaga T, and Kanehisa H. Influence of tendon slack on electromechanical delay in the human medial gastrocnemius in vivo. J Appl Physiol (1985) 96: 540-544, 2004.

28. Nordez A, Gallot T, Catheline S, Guevel A, Cornu C, and Hug F. Electromechanical delay revisited using very high frame rate ultrasound. J Appl Physiol (1985) 106: 1970-1975, 2009.

29. Périard JD, Racinais S, and Thompson MW. Adjustments in the force-frequency relationship during passive and exercise-induced hyperthermia. Muscle Nerve 50: 822-829, 2014.

30. Point M, Guilhem G, and Hug F. Cryotherapy induces an increase in muscle stiffness. 28: 260-266, 2018.

31. Racinais S, Gaoua N, and Grantham J. Hyperthermia impairs short-term memory and peripheral motor drive transmission. J Physiol 586: 4751-4762, 2008.

32. Racinais $\mathbf{S}$ and Girard $\mathbf{O}$. Neuromuscular failure is unlikely to explain the early exercise cessation in hot ambient conditions. Psychophysiology 49: 853-865, 2012.

33. Racinais S and Oksa J. Temperature and neuromuscular function. Scand J Med Sci Sports 20 Suppl 3: 1-18, 2010.

34. Racinais S, Wilson MG, and Périard JD. Passive heat acclimation improves skeletal muscle contractility in humans. Am J Physiol Regul Integr Comp Physiol 312: R101-r107, 2017.

35. Ramanathan NL. A new weighting system for mean surface temperature of the human body. J Appl Physiol 19: 531-533, 1964.

36. Ranatunga KW. Temperature-dependence of shortening velocity and rate of isometric tension development in rat skeletal muscle. J Physiol 329: 465-483, 1982.

37. Rutkove SB. Effects of temperature on neuromuscular electrophysiology. Muscle Nerve 24: 867-882, 2001.

38. Rutkove SB, Kothari MJ, and Shefner JM. Nerve, muscle, and neuromuscular junction electrophysiology at high temperature. Muscle Nerve 20: 431-436, 1997.

39. Sapin-de Brosses E, Gennisson JL, Pernot M, Fink M, and Tanter M. Temperature dependence of the shear modulus of soft tissues assessed by ultrasound. Phys Med Biol 55: 1701-1718, 2010.

40. Schertzer JD, Green HJ, and Tupling AR. Thermal instability of rat muscle sarcoplasmic reticulum $\mathrm{Ca}(2+)$-ATPase function. Am J Physiol Endocrinol Metab 283: E722-728, 2002.

41. Segal SS, Faulkner JA, and White TP. Skeletal muscle fatigue in vitro is temperature dependent. J Appl Physiol (1985) 61: 660-665, 1986.

42. Stein RB, Gordon T, and Shriver J. Temperature dependence of mammalian muscle contractions and ATPase activities. Biophys J 40: 97-107, 1982.

43. Todnem K, Knudsen G, Riise T, Nyland H, and Aarli JA. The non-linear relationship between nerve conduction velocity and skin temperature. J Neurol Neurosurg Psychiatry 52: 497-501, 1989. 
44. Walker P, Amstutz HC, and Rubinfeld M. Canine tendon studies. II. Biomechanical

45. Wang JC, Kabo JM, Tsou PM, Halevi L, and Shamie AN. The effect of uniform heating on the biomechanical properties of the intervertebral disc in a porcine model. Spine J 5: 64-70, 2005.

46. Zhou S, Carey MF, Snow RJ, Lawson DL, and Morrison WE. Effects of muscle fatigue and temperature on electromechanical delay. Electromyogr Clin Neurophysiol 38: 67-73, 1998. 


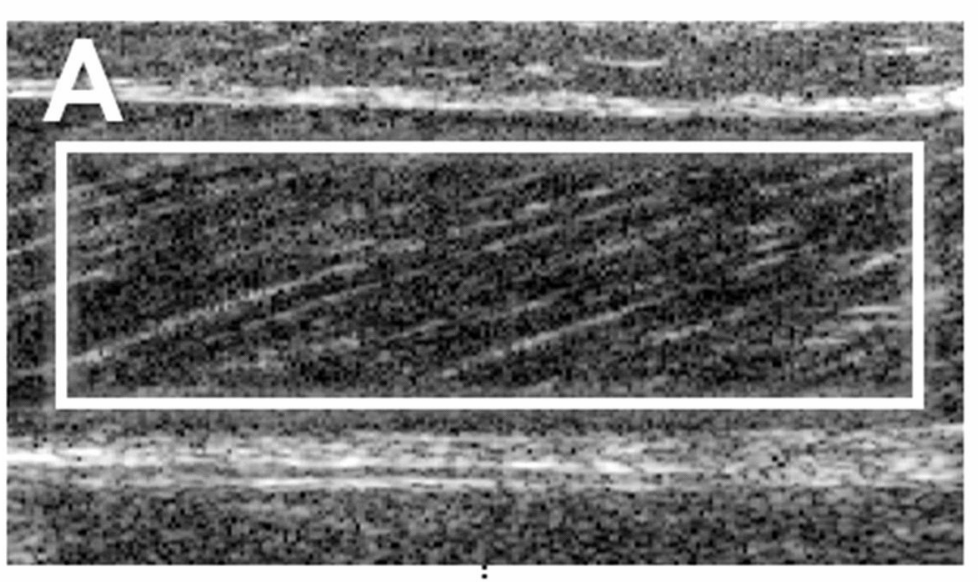

Muscle

C stimulation

Muscle fascicle motion

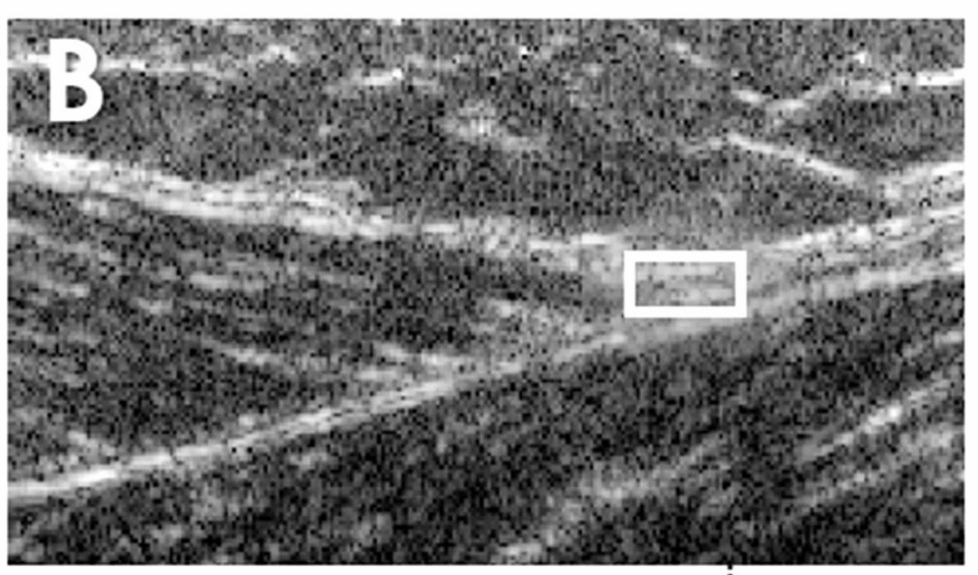

Myotendinous junction motion
Force production

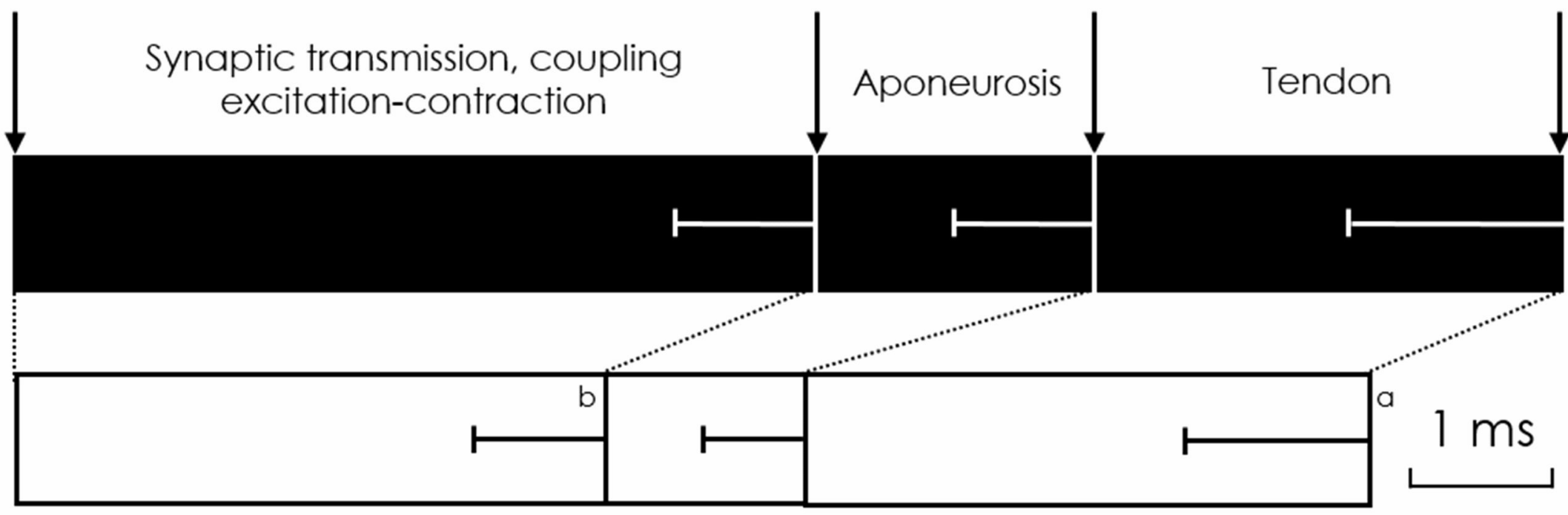

$\mathrm{CON}$ excitation-contraction 
Table 1. Main characteristics of the nervous electrically-evoked twitch in control ambient (CON) and hot ambient (HOT) environment.

\begin{tabular}{lcccc}
\hline & CON & HOT & $\boldsymbol{P}$ & $\boldsymbol{\eta}^{\mathbf{2}}$ \\
\hline PT (N) & $66.9 \pm 12.9$ & $71.8 \pm 13.9$ & 0.301 & 0.036 \\
CT (ms) & $121.2 \pm 7.5$ & $110.2 \pm 5.7^{* * *}$ & $<0.001$ & 0.419 \\
HRT (ms) & $88.8 \pm 6.1$ & $75.4 \pm 8.1^{* * *}$ & $<0.001$ & 0.485 \\
RFD (N.ms & & $\left.0.65 \pm 0.11^{*}\right)$ & 0.029 & 0.149 \\
\hline
\end{tabular}

Values are presented as mean $\pm \mathrm{SD}$. PT, peak twitch amplitude; CT, contraction time; HRT, half relaxation time; RFD, rate of force development. * Significant difference between HOT and CON, $P<$ $0.05 ; * * *, P<0.001$. 